\title{
ORIGINAL ARTICLE Genetic basis of stage-specific melanism: a putative role for a cysteine sulfinic acid decarboxylase in insect pigmentation
}

\author{
SV Saenko ${ }^{1,3}$, MA Jerónimo ${ }^{2}$ and P Beldade ${ }^{1,2}$ \\ Melanism, the overall darkening of the body, is a widespread form of animal adaptation to particular environments, and includes \\ bookcase examples of evolution by natural selection, such as industrial melanism in the peppered moth. The major components \\ of the melanin biosynthesis pathway have been characterized in model insects, but little is known about the genetic basis of \\ life-stage specific melanism such as cases described in some lepidopteran species. Here, we investigate two melanic mutations \\ of Bicyclus anynana butterflies, called Chocolate and melanine, that exclusively affect pigmentation of the larval and adult \\ stages, respectively. Our analysis of Mendelian segregation patterns reveals that the larval and adult melanic phenotypes are due \\ to alleles at different, independently segregating loci. Our linkage mapping analysis excludes the pigmentation candidate gene \\ black as the melanine locus, and implicates a gene encoding a putative pyridoxal phosphate-dependant cysteine sulfinic acid \\ decarboxylase as the Chocolate locus. We show variation in coding sequence and in expression levels for this candidate larval \\ melanism locus. This is the first study that suggests a biological function for this gene in insects. Our findings open up exciting \\ opportunities to study the role of this locus in the evolution of adaptive variation in pigmentation, and the uncoupling of \\ regulation of pigment biosynthesis across developmental stages with different ecologies and pressures on body coloration. \\ Heredity (2012) 108, 594-601; doi:10.1038/hdy.2011.127; published online 11 January 2012
}

Keywords: evo-devo; pigmentation; lepidoptera; melanogenesis; PLP-dependent enzyme; black

\section{INTRODUCTION}

One of the most exciting questions in biology is how the diversity of living forms has evolved. Animal coloration is a visually compelling example of such diversification. It is an adaptive trait that plays a role not only in visual communication (for example, interactions with predators and mates), but also in thermoregulation, UV protection and pathogen resistance (True, 2003; Caro, 2005). Variation in coloration is common in natural populations and recently has become a foremost model in studies of the interactions between evolutionary and developmental processes that shape phenotypic variation and diversification (Protas and Patel, 2008; Mills and Patterson, 2009; Wittkopp and Beldade, 2009).

In the past decades, evo-devo studies of animal coloration have advanced our understanding of the genetic mechanisms underlying adaptive changes in overall body pigmentation, and in the color patterns formed by the spatial organization of pigmented cells. Multiple signaling pathways, transcription factors and enzymes have been associated with the evolution of color patterns in vertebrates (Mills et al., 2007; Manceau et al., 2011) and arthropods (Wittkopp et al., 2002; Gompel et al., 2005). Some of those have also been implicated in changes in overall body coloration, including dramatic reduction of pigmentation in animals inhabiting caves (Gross et al., 2009; Protas et al., 2011) or light background environments (Rosenblum et al., 2010), as well as dramatic darkening of the body, or melanism, which is addressed here.
Naturally occurring melanic phenotypes are widespread in arthropods and vertebrates as an adaptation for life in particular environments. The genetic basis of melanism has been investigated in different systems where black coloration renders animals cryptic in relatively dark backgrounds; for example, in pocket mice living on lava flows (Nachman et al., 2003), in night-hunting cats (Eizirik et al., 2003) and in the peppered moth example of industrial melanism (Van't Hof and Saccheri, 2010; Van't Hof et al., 2011). The biosynthesis of melanin and other tyrosine-derived pigments, the relative levels of which determine the color of the vertebrate skin and hair and of the insect cuticle, has been studied extensively in model organisms (Protas and Patel, 2008). A number of melanogenesis genes have been associated with larval and adult melanic phenotypes in the insect genetic models Drosophila melanogaster and Bombyx mori (Wittkopp et al., 2002; True et al., 2005; Zhan et al., 2010). These genes encode enzymes involved in melanin biosynthesis and are obvious candidates for harboring allelic variation contributing to naturally occurring variation in body pigmentation.

Among insects, Lepidoptera (the order of butterflies and moths) show spectacular adaptive variation in color of larval and pupal integument (Hazel, 2002; Jones et al., 2007; Noor et al., 2008), and in adult wing patterns (Beldade and Brakefield, 2002; Saenko et al., 2011). Caterpillars and adults typically exploit different habitats, with specific ecological challenges and selective pressures on their pigmentation. In some cases, stage-specific ecological pressures can lead to

${ }^{1}$ Institute of Biology, Leiden University, Leiden, The Netherlands and ${ }^{2}$ Instituto Gulbenkian de Ciência, Oeiras, Portugal

${ }^{3}$ Current address: Department of Genetics \& Evolution, University of Geneva, Geneva, Switzerland.

Correspondence: Dr P Beldade, Instituto Gulbenkian de Ciência, Rua da Quinta Grande 6, Oeiras P-2780-156, Portugal.

E-mail: pbeldade@igc.gulbenkian.pt or Dr SV Saenko, Department of Genetics \& Evolution, University of Geneva, Quai Ernest-Ansermet 30,1211 Geneva 4, Switzerland.

E-mail: siouzanna.saenko@unige.ch

Received 20 August 2011; revised 25 October 2011; accepted 17 November 2011; published online 11 January 2012 
variation in pigmentation even between larval instars (for example, white-brown early instars of Papilio xuthus butterflies mimic bird droppings, whereas the last instar has a green camouflage color; Futahashi and Fujiwara, 2005), or between different wing surfaces of adult butterflies (for example, dorsal and ventral color patterns in Bicyclus anynana butterflies involved in mate choice or in predator avoidance, respectively, Oliver et al., 2009). The diversified pigmentation phenotypes of different life-stages of lepidopteran species provide a good model for studies of the development and evolution of stagespecific color and color patterns.

The availability of mutants with altered pigmentation or color patterns is especially useful for dissecting the genetic basis of variation in coloration (for example, Weatherbee et al., 1999; Koch et al., 2000; Futahashi et al., 2010; Saenko et al., 2010). Here, we focus on two spontaneous melanic mutations of the nymphalid butterfly $B$. anynana, a lab model for studies of wing pattern evolution and development (Beldade and Brakefield, 2002; Beldade et al., 2005; Conceição et al., 2011) and of developmental plasticity (Beldade et al., 2011). Both mutant alleles lead to overall darkening of the body, but while one (named Chocolate) affects exclusively the larval stages, the other (named melanine) affects only adult pigmentation (Figure 1). We describe the Mendelian segregation of both alleles and show that the larval and adult melanism are not due to genetic variation at the same locus. Furthermore, we provide a detailed genetic investigation of the larval melanism locus, with characterization of sequence and

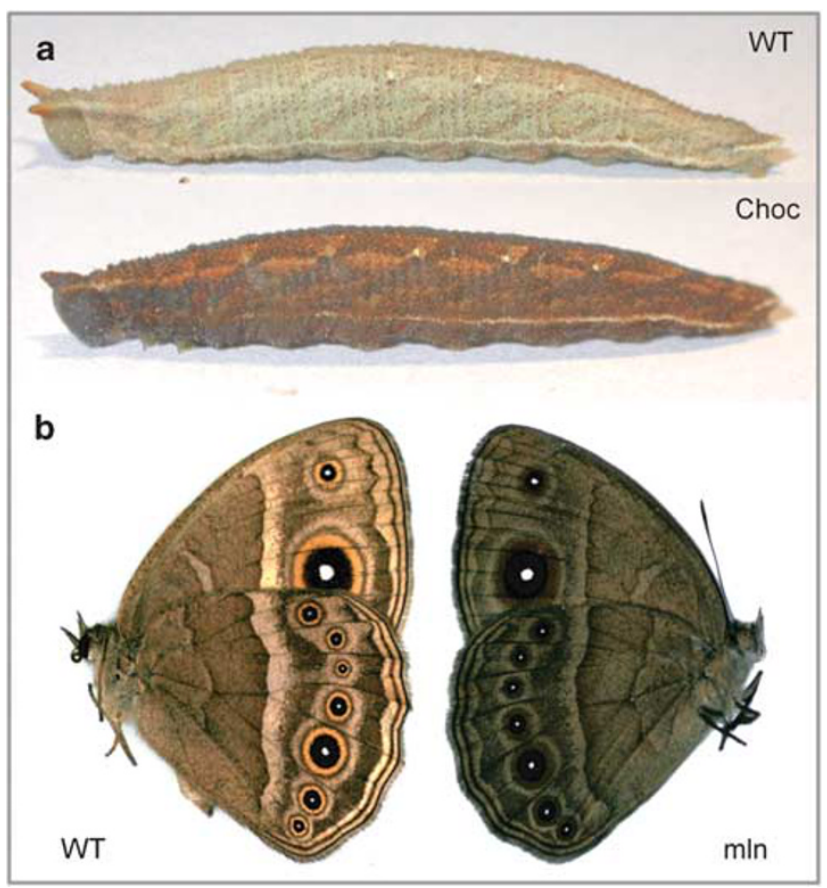

Figure 1 Stage-specific melanic mutants of Bicyclus anynana. (a) Lateral view of the mid-last instar "WT" and Choc larvae. Typical color of WT head capsule and body integument is light brown, whereas that of the Choc mutants is dark reddish brown (pupal and adult coloration are not affected). The effect of the Choc allele is visible but much weaker in earlier larval instars; the dark coloration in these disappears almost completely by the end of each instar, just before each molt. (b) Ventral view of WT and melanine $(\mathrm{m} / \mathrm{n})$ adult females. Typical adult coloration is brown with light bands and concentric rings of white, black and yellow running in the anterior-posterior direction along the wings. Homozygous adults for the $\mathrm{m} / \mathrm{n}$ allele are overall dark, almost black (larval and pupal coloration are not affected). expression differences in pigmentation genes. This study suggests a role in body pigmentation for a gene whose orthologs in other insects have not yet been associated with any biological process.

\section{MATERIALS AND METHODS}

\section{Experimental animals and candidate genes}

The Chocolate (Choc) and melanine ( $\mathrm{mln}$ ) stocks of B. anynana (for clarity, we refer to phenotypes in regular font and to genotypes in italics) were each originally set-up from single individuals isolated from a 'wild-type' (WT) lab population (Brakefield et al., 2009), and since maintained with selection in favor of the mutant phenotype. To preserve the health of mutant stocks facing potential effects of inbreeding depression or disease, these can be occasionally outcrossed with healthy WT individuals. All animals in this study were reared at $27^{\circ} \mathrm{C}$ as described before (Brakefield et al., 2009).

The complete list of $B$. anynana candidate genes analyzed in this study (different subsets in the linkage and expression analyses described below) include those encoding key enzymes in melanogenesis (pale, Ddc, black, tan, yellow, ebony and purple) and one suggested by previous linkage mapping work (CSAD, details below). The B. anynana orthologs of these genes were derived from the species' EST collection available on GenBank (Beldade et al., 2006, 2009), as explained below for each of the different cases.

\section{Cloning and phylogenetic analysis of $B a \_b l a c k$ and $B a \_C S A D$}

Candidate genes black (encoding an enzyme involved in melanin synthesis) and CSAD (encoding cysteine sulfinic acid decarboxylase) were chosen based on function in pigmentation in other insects or position in the sequenced genome of the reference lepidopteran B. mori (see below), respectively. B. anynana ESTs corresponding to these candidate genes were identified by BLAST-screening the NCBI EST database with the predicted protein sequences of $D$. melanogaster black (FlyBase; http://flybase.org/) and B. mori BGIBMGA010122 (Silkworm Genome Database; http://silkworm.genomics.org.cn/). The full coding sequences of $B a \_b l a c k$ and $B a_{-} C S A D$ were then obtained with regular PCR or rapid amplification of cDNA ends (RACE), respectively.

Two B. anynana ESTs with the highest similarity to D. melanogaster gene black (FlyBase: CG7811), GE718264 (tblastn e-value $=1 \mathrm{e}-59,59 \%$ identities) and GE718255 (tblastn e-value $=5 \mathrm{e}-59,64 \%$ identities), correspond to the $5^{\prime}$ and $3^{\prime}$ regions of $B a \_b l a c k$, respectively. The full coding sequence of this gene was obtained by PCR with primers $5^{\prime}$-ACGTTGCACGCTATTCAGTG- $3^{\prime}$ and $5^{\prime}$-CTGCCATAAACGCCAGAAG-3' on cDNA prepared from total RNA (extracted from whole cuticle of last instar larvae using Trizol, Invitrogen, Paisley, UK) with Reverse Transcription System (Promega, Leiden, The Netherlands). B. anynana EST GE676169, that with the highest similarity (tblastn e-value $=2 \mathrm{e}-54,68 \%$ identities) to $B$. mori predicted protein BGIBMGA010122 (Silkworm Genome Database), corresponds to the $3^{\prime}$ region of $B a_{-} C S A D$. Its full coding sequence was obtained by RACE PCR with primers $5{ }^{\prime}$-CGCTAC CATTGCTTGAGATCGCAGTG- $3^{\prime}$ and $5^{\prime}$-AACTTCCACCCCAACAAGCATC GACA-3' on cDNA prepared from total RNA (extracted as above) with SMART RACE Amplification Kit (Clontech, Saint-Germain-en-Laye, France). The nucleotide sequences of $B a \_C S A D$ and $B a \_b l a c k$ complete coding regions were deposited to GenBank under accession numbers JN003848 and JN003850, respectively.

Orthologs of both genes from other insects were identified using BLASTp in NCBI (that is, top hits for different species with e-value below e-150) and aligned with Multiple Sequence Comparison by Log-Expectation (MUSCLE) tool (http://www.ebi.ac.uk/Tools/). A phylogenetic tree was generated using the neighbour-joining method with the bootstrap analysis in the MEGA5 program (http://www.megasoftware.net/).

\section{Mapping families and linkage analysis}

To determine whether mutations responsible for Choc and mln phenotypes occurred in the same gene, reciprocal crosses between one Choc and one mln homozygous individuals were set-up: two crosses between a Choc male and a mln female and two crosses between a mln male and a Choc female (Figure 2a). From each of the four F1 families, two males were crossed to their sisters to obtain eight F2 families with a total of 259 individuals (details in Supplementary Table S1). All these were phenotyped for larval (Choc vs WT; Figure 1a) 
a

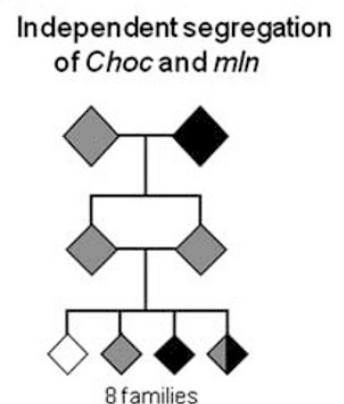

\begin{tabular}{c|c|c|} 
& $W T$ & mln \\
\hline$W T$ & 40 & 23 \\
\hline Choc & 141 & 55 \\
\hline
\end{tabular}

b

Independent segregation of $\mathrm{m} / \mathrm{n}$ and black

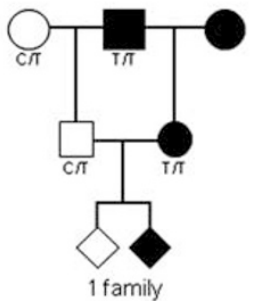

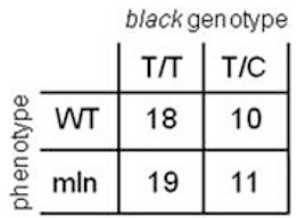

C

Tight linkage between Choc and Ba_CSAD
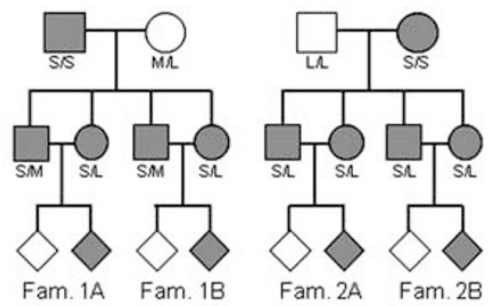

Figure 2 Analysis of linkage of melanism loci. Top: schematic representations of the experimental crosses used to test for linkage between loci responsible for larval Choc and adult mln phenotypes (a), between $\mathrm{m} / \mathrm{n}$ and candidate gene Ba_black (b), and between Choc and candidate gene Ba_CSAD (c). Different phenotypes are represented by color: gray for Choc melanic larvae (with WT-colour adults), black for mln melanic adults (with WT-colour larvae), and white for WT larval and adult coloration. Males are shown as squares, females as circles and diamonds represent offspring of both sexes. Bottom: tables showing the numbers of offspring in each phenotypic/genotypic class (details about the mapping families in Supplementary Tables S1 and S2). DNA sequence polymorphisms used to genotype the individuals in (b) and (c) are explained in the text.

and adult (mln vs WT; Figure $1 \mathrm{~b}$ ) pigmentation. The numbers of progeny in each phenotypic class were pooled over the eight families, and totals were tested against the numbers expected under the hypothesis that the loci responsible for the larval and the adult melanic phenotypes are not the same (that is, that the Choc and $m l n$ alleles segregate independently) using the $\chi^{2}$ goodness-of-fit test.

For the analysis of co-segregation between the mln phenotype and variation at candidate gene Ba_black (explained in the Results and Discussion section), a single backcross panel consisting of $30 \mathrm{mln}$ and 28 WT individuals was obtained by crossing one heterozygote male (offspring of a cross between a $\mathrm{mln}$ and a WT butterfly) to one mln female (Figure $2 \mathrm{~b}$ ). All butterflies were phenotyped and genomic DNA was extracted from thoraces using the DNeasy Tissue Kit (Qiagen, Hilden, Germany), following manufacturer's recommendations. A 260-bp fragment of the coding sequence of candidate gene Ba_black was amplified and sequenced in the grandparents with primers $5^{\prime}$-ACGTTG CACGCTATTCAGTG-3' and 5'-AATGGCGTATCCGTTAGCAA-3'. A singlenucleotide polymorphism (SNP), C/T in position 119 of the amplified fragment (corresponding to position 381 in the coding sequence of JN003850), is located within the recognition site for the endonuclease AccI, which cuts the fragments only in the presence of C. Genotyping assays based on this polymorphism were carried out in the parents and $58 \mathrm{~F} 2$ offspring of the mapping family as follows: the fragments of Ba_black were amplified with the same primers as above, digested overnight with $5 \mathrm{U}$ AccI (New England Biolabs, Ipswich, USA) following the manufacturer's recommendations, and scored on agarose gels.

For the analysis of co-segregation between the Choc phenotype and variation at candidate gene $B a_{-} C S A D$ (explained in the Results and Discussion section), each of two Choc individuals were crossed to a WT butterfly from an unrelated laboratory population, and an F2 mapping panel consisting of 264 Choc and 85 WT individuals was established (details in Figure $2 \mathrm{c}$ and Supplementary Table S2). Genomic DNA for individuals in this panel was obtained as described above. Fragments of the candidate gene $B a_{-} C S A D$ were amplified in the grandparents with primers $5^{\prime}$-ATTGACGCGTTCAAACTGTG- $3^{\prime}$ and 5'-ACAAGACCTGGAATTTCCCTA- $3^{\prime}$, cloned and sequenced to confirm PCR specificity. We identified a length polymorphism in the last intron of the gene, discriminating between the grand-parental alleles: 1086-bp allele in the Choc individuals, and a 1189-bp plus a 1305-bp alleles in the WT individuals. A genotyping assay based on this length polymorphism was carried out in the eight parents and all 349 F2 offspring of the four families by amplifying fragments of $B a \_C S A D$ with the same primers as above and by scoring allele lengths on agarose gels.

\section{Sequence and expression variation in candidate genes}

For the analysis of coding sequence variation in $B a_{-} C S A D$, primers 5'-GAACCACACGTTCGAATTTCA- $3^{\prime}$ and 5'-ACAAGACCTGGAATTTCCCTA- $3^{\prime}$ were used to amplify a 1592 -bp fragment (base pairs $46-1637$ in JN003848, Figure 3a) including the gene's open reading frame from cDNA (prepared from larval cuticle, as above) of four WT and four Choc offspring of a cross between a Chocl+ male and a WT female. The products were cloned into the pCRII-TOPO vector (Invitrogen), and five clones per individual were Sanger-sequenced with M13 primers in both directions. All sequences were aligned in BioEdit to identify SNPs and indels.

For the analysis of expression variation, we performed semi-quantitative PCR on cDNA representing transcripts in different stages of larval integument development in individuals from the WT and the pure-breeding Choc stocks. Using pure-breeding stocks, rather than melanic and non-melanic siblings of a single cross, allows us to be certain of individual genotypes even at stages when phenotypes are not obvious. Six individuals were dissected for each of four developmental stages, characterized by the following criteria: (1) late 4th instar (4-5 days after the third molt), (2) pre-molt (next-instar head capsule is visible through the cuticle, which becomes lighter), (3) 1st day of the 5th instar (18-24h after the 4 th molt), (4) 2 nd day of the 5 th instar $(42-48 \mathrm{~h}$ after the 4 th molt). The integument was dissected from segments $1-5$ of the abdomen, and muscles and fat tissue attached to the epidermis were removed. Total RNA was extracted as described above, and $200 \mathrm{ng}$ of RNA of each individual was used to prepare cDNA with Reverse Transcription System (Promega) and oligo(dT) primers, following manufacturer's instructions. Expression levels of $B a \_C S A D$, $B a \_b l a c k$ and six genes with known role in melanin biosynthesis were examined with semi-quantitative PCR using primers that amplify fragments, which span introns, to avoid noise due to potential residual contamination of cDNA with genomic DNA (see Supplementary Table S3 for GenBank accession numbers and primer sequences). During protocol optimization, negative controls were performed on water, and, to establish the optimal number of cycles, each primer pair was tested with 25,30 and 35 PCR cycles on a mix of cDNA prepared from all WT individuals. The amplicons were also sequenced to confirm gene identity. The housekeeping gene FK506 binding protein 2 (FK506) was used as an internal control (Pijpe et al., 2011). PCRs were performed on 
Ba CSAD

BGI $\mathrm{IM} G A 010122$

$\mathrm{Ba}$ CSAD

BGI $\bar{I}$ MGA 010122

Ba_CSAD

BGI $B M G A 010122$

Ba CSAD

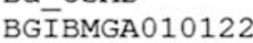

Ba CSAD

BGI IBMGA010122

Ba_CSAD

BGI BMGA010122

Ba CSAD

BGI $\bar{I} B$ GA 010122

Ba_CSAD

BGI $\mathrm{IM} G A 010122$

Ba_CSAD

BGI IMMAA 010122
MELNGHTFLDKVLGIVKEETDHETALIQFKHPKELEEILRENLDISKKLNDQDLEKAVRK 60 MDLS---FLDRVLQIVKDERKKDVPLVREKHPEELEAIL--DLDIGQEVNDDDLERCVRQ 55

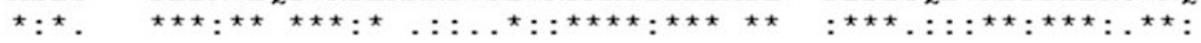

VLQYSVKTNKPTFRNQLYGGTDPYGLAGAWIAEAFNTSQYTFEVAPVFTLIELKVLDHIL 120 VLQYSVKTDKATFKNQLYGCTDPYGLAGAWIAEAFNTSQYTFEVAPVFTLIELKVLNHIL 115

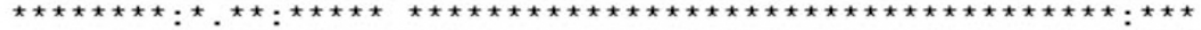

KLFGISDGDGIFSPGGSISMLYALVAAASKAFPEVKRIGMGGLPEMVIVTSEDSHYSILK 180 NLFGIPNGDGIFSPGGSVSMLYALVAARFKAFPEVKRKGMRNLPEMVIFTSEDSHYSIRK 175

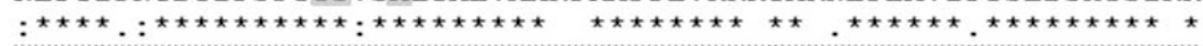

AAHWLGEGIENVKVIKTNDHGQMSATDLENTLQQELALGRTPLMVNATAGTTVLGAIDDL 240 AAHWLGFGTESLRPIKANEHGQMIVSELEKAIEREKKENRYPVLVNATAGTTVLGAIDDL 235

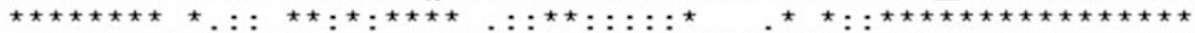

ENVAAICEKYGVWMHVDACWGGSLILSKKYRSKLKGINRASSISWNPHKMMGVPLQCSVF 300 NDIANVCKKHGVWMHVDACWGGSLMLSPKYRSKLLGVKRADSISWNPHKMAGAPLQCSVF 295

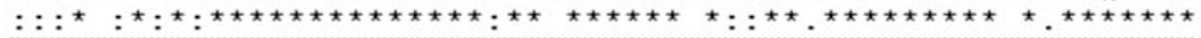

LLREKGVLHEANSAAAQYLFQQDKFYDVSYDTGDKSIQCGRKIDAFKLWMIWKARGDIGL 360 LIKERGLLHEANSAAAOYLEQQDKFYDVSYDTGDKSVQCGRKIDAFKLWMMWKSRGDIGL 355

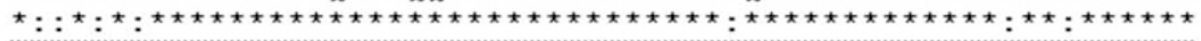

NHLTDHVMEIAEFCIQTVAHRSNFRLVTMHMQCPNVCEWYI PT FMNNKMENEEWWNTTHK 420 SVLTDKVMSIAQFCLSATSKMEGERLVSEKLQCPNVCFWYI PKFMRNKDENEEWWDLMHK 415

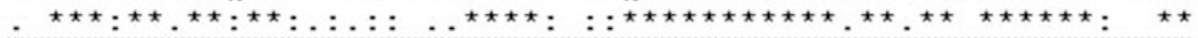
ITPKIKEQLTLRSQAMVAYSPLRERKNFFRLAFTFHPVLEKSHVLDILQAIEECGEGITL 480 ITPKIKEQLTLTSKLMVAYSPLRQHKNFFRLAFTFHPELNENQVIEMLNAVKLSGEAITK 475

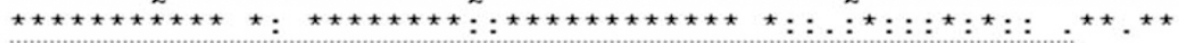

PVL- 483

EMFP 479

: :

b

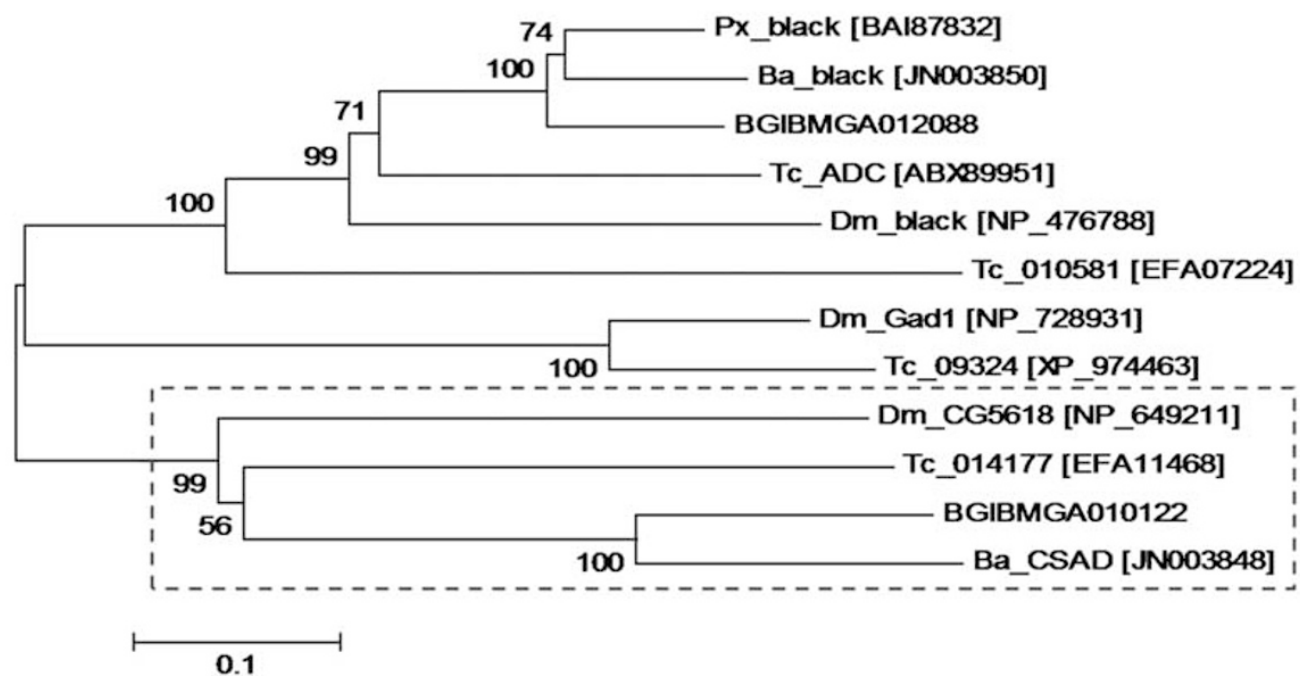

Figure 3 Sequence annotation and phylogenetic analysis of Ba_CSAD. (a) Alignment of the predicted protein sequences of Ba_CSAD (483 amino acids) and its silkworm ortholog BGIBMGA010122 (479 amino acids). Numbers on the right show amino acid sequence position; sequence identities are marked with $\left({ }^{*}\right)$, conserved substitutions with $(:)$ and semi-conserved substitutions with (.) (cf. MUSCLE, see Materials and methods). Dashed line indicates the PLPdependent DOPA decarboxylase domain, gray boxes indicate pyridoxal 5'-phosphate-binding residues and the catalytic lysine (cf. NCBI Conserved Domains Search; see Materials and methods), and the arrow indicates the threonine, which is substituted by lysine in the mutant allele. (b) Neighbor-joining phylogenetic tree of $\mathrm{Ba}$ CSAD and related insect proteins based on their amino acid sequences (with corresponding GenBank accession numbers), with bootstrap values for 1000 replications. Ba_CSAD, B. mori BGIBMGA010122, T. castaneum 014177 and D. melanogaster CG5618 form a separate clade, indicated with the dashed square. The scale bar indicates the evolutionary distance between the groups. 

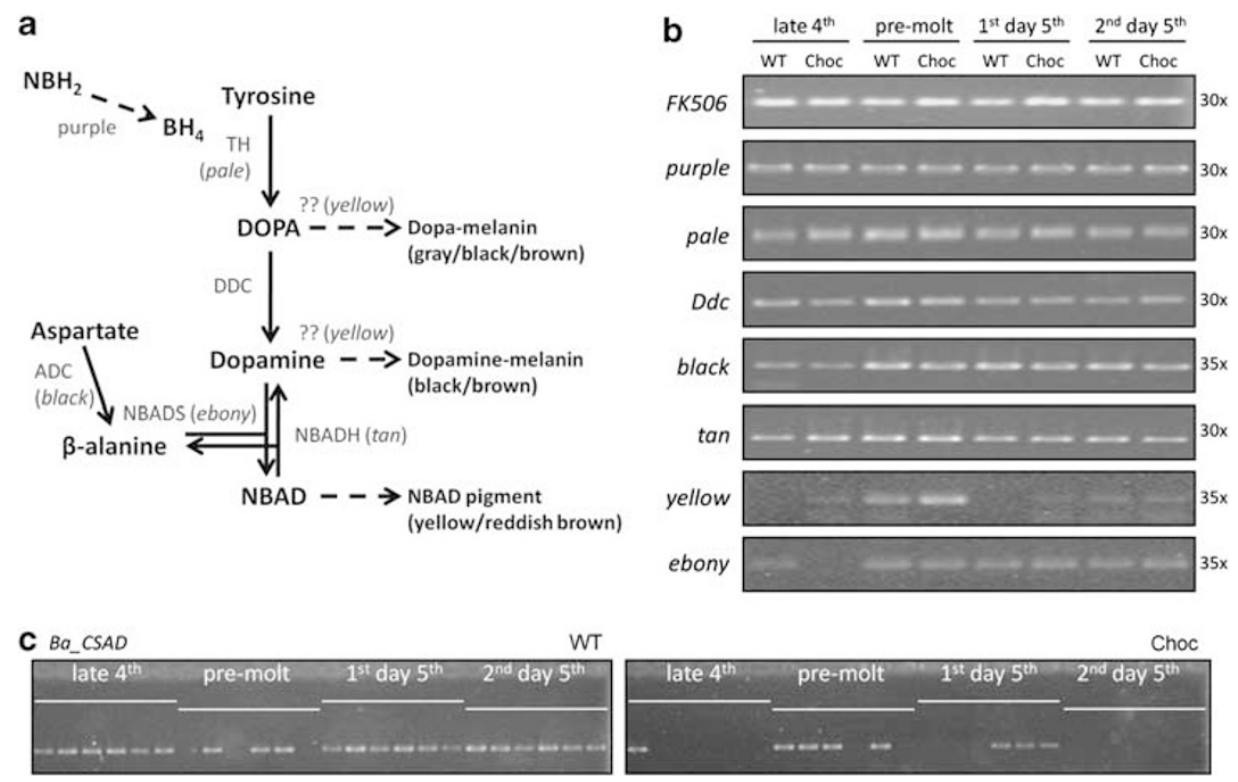

Figure 4 Melanin pathway and semi-quantitative PCR of key genes in WT and Choc larvae. (a) A simplified schematic representation of the melanin pathway (adapted from Arakane et al., 2009; Zhan et al., 2010; Futahashi et al., 2010): enzymes are shown in gray with corresponding genes in brackets; arrows indicate reactions catalyzed by each enzyme (dashed arrows correspond to multi-step reactions). TH, tyrosine hydrolase; putative DCE, dopachrome conversion enzyme (note that the exact function of the yellow gene is still unclear); DOPA decarboxylase (DDC), dopa decarboxylase; ADC, aspartate decarboxylase; NBADS, N-beta-alanyldopamine synthase; NBADH, N-beta-alanyldopamine hydrolase; $\mathrm{NBH}_{2}$, dihydroneopterin; $\mathrm{BH}_{4}$, tetrahydrobiopterin. (b) Relative expression levels of the control FK506 and seven melanin pathway genes in larval integument around the last larval molt (stages indicated on top). PCR reactions (number of cycles on the right) were run on six replicate individuals per stage per phenotype (Supplementary Figure S1) and, for illustration, also on pools of replicates (shown here). (c) Semi-quantitative analysis of Ba_CSAD expression in WT and Choc larvae, performed at 35 cycles on six individuals for each stage and phenotype (data for 30 and 38 cycles is shown in Supplementary Figure S1). The fact that PCRs worked for both phenotypic groups at some stage assures that lack of amplification is unlikely to be caused by putative sequence differences between populations.

cDNA of each individual and, for illustration in Figure 4b, on pools of $5 \mu \mathrm{l}$ cDNA of individuals belonging to the same stage and phenotypic class. The PCR conditions were: $95^{\circ} \mathrm{C}$ for $3 \mathrm{~min}$, followed by 30 and/or 35 (and also 38 for $\mathrm{Ba} \_\mathrm{CSAD}$ ) cycles of $94^{\circ} \mathrm{C}$ for $20 \mathrm{~s}, 60^{\circ} \mathrm{C}$ for $20 \mathrm{~s}, 72^{\circ} \mathrm{C}$ for $20 \mathrm{~s}$ and a final extension at $72^{\circ} \mathrm{C}$ for $5 \mathrm{~min}$. Differences in expression were assessed by running $5 \mu \mathrm{l}$ of PCR products on $1.2 \%$ agarose gels.

\section{RESULTS AND DISCUSSION}

\section{Mutations in two different loci cause melanism at different} developmental stages

The color of the integument of young B. anynana larvae is typically green and changes to light brown in the final (fifth) instar; the adults are largely brown with more or less conspicuous wing patterns (Figure 1). Although there is subtle quantitative variation for different aspects of larval and adult coloration (Beldade et al., 2002; Allen et al., 2008), particular alleles can have very large effects and produce phenotypes well outside the normal distribution range (Beldade et al., 2008, Saenko et al., 2008; Saenko et al., 2010). This is the case for two spontaneous mutations which affect overall pigmentation, each at a specific developmental stage. The recessive $m l n$ and the dominant Choc mutations cause dramatic darkening of adults and larvae, respectively, without having any detectable effects during other developmental stages (Figure 1). Note that the Choc phenotype is virtually indistinguishable from that caused by the $B$. anynana dark larvae allele described elsewhere (Bear et al., 2010), but, while the latter is recessive, the Choc allele we investigate is dominant (Beldade et al., 2009). Because the locus carrying the dark larvae allele was not mapped, we cannot establish if the two mutations are at the same locus.
As the Choc and $m l n$ mutations cause overall darkening of the integument, we proceeded to investigate whether they correspond to alleles of the same or different genes. We performed crosses between homozygotes for either of the two mutant alleles and investigated the frequency of different phenotypes in $259 \mathrm{~F} 2$ progeny of eight families (Figure 2a and Supplementary Table S1). The total number of progeny in each phenotypic class was not different from that expected if Choc and $m \ln$ are alleles of two independently segregating loci (goodnessof-fit chi-square test $=5.62$, d.f. $=3, P=0.87$ ). Moreover, 40 out of 259 individuals had WT coloration at both larval and adult stages, which is only possible if Choc and $m \ln$ are not alleles at the same locus and can, therefore, complement.

\section{Analysis of linkage between mutant loci and candidate genes}

Several genes have been associated with melanization of the whole body or specific body parts in insects (True, 2003; Wittkopp and Beldade, 2009). In particular, loss-of-function mutations in the gene black (encoding Aspartate 1-decarboxylase, involved in production of melanin substrate beta-alanine, Kramer et al., 1984) result in overall darkening of adult fruitflies (Phillips et al., 2005) and beetles (Arakane et al., 2009), a phenotype that resembles that of B. anynana mln mutants. Hence, we performed linkage analysis between the $m l n$ allele, whose location in the $B$. anynana genome is unknown, and a polymorphic marker in Ba_black, previously mapped to $B$. anynana linkage group (LG) 11 (Beldade et al., 2009), in a single backcross family consisting of $28 \mathrm{WT}$ and $30 \mathrm{mln}$ offspring (Figure $2 \mathrm{~b}$ ). We identified a synonymous $\mathrm{C} / \mathrm{T}$ polymorphism in the coding sequence of Ba_black in the grandparents and genotyped the parents and the 58 offspring (see Materials and methods). In case of tight linkage between 
$B a \_b l a c k$ and the locus responsible for the mutant phenotype, mln offspring would be expected to carry only the T allele at the Ba_black locus (like their mln grandfather), while WT offspring should have the $\mathrm{C} / \mathrm{T}$ genotype (like their WT grandmother). However, we found that 11 out of $30 \mathrm{mln}$ individuals had C/T and 18 out of $28 \mathrm{WT}$ individuals had T/T genotypes (Figure $2 \mathrm{~b}$ ), which is close to $50 \%$ recombination rate between $\mathrm{Ba}$ black and the locus carrying the $m \ln$ mutation. This indicates that the two loci are either on different chromosomes or at a large distance on the same chromosome, and that $\mathrm{mln}$ is not an allele of Ba_black.

The Choc allele was previously mapped to a $35 \mathrm{cM}$ region on B. anynana LG 7 (Beldade et al., 2009). This excludes Ba_black, mapped to LG 11, and other melanin pathway genes found on other LGs in B. anynana (for example, Ddc on LG 4, Beldade et al., 2009), and in the syntenic reference lepidopteran genome of the silkworm B. mori (for example, ebony on LG 26, Futahashi et al., 2008). The region of the silkworm genome orthologous to the $B$. anynana genomic region where Choc maps to was identified as nscaf2986 (Beldade et al., 2009 see Supplementary Table S4 for gene content of this B. mori genomic region). This region contains the gene BGIBMGA010122, which shows high sequence similarity to D. melanogaster gene CG5618 (encoding a predicted cysteine sulfinic acid decarboxylase, CSAD; NCBI tblastn evalue 3e-156, 49\% identities), gene CG14994 (encoding a glutamic acid decarboxylase 1, GAD1; e-value 2e-123, 42\% identities) and gene black (e-value 1e-147, 45\% identities). As Aspartate-1 decarboxylase (the product of black) is involved in cuticular melanization in other insects (see above), we wished to investigate whether the $B$. anynana ortholog of BGIBMGA010122 was responsible for melanization of the larval integument in the Choc mutant.

We identified the B. anynana EST with the highest similarity to BGIBMGA010122 (GE676169, see Materials and methods) and carried out linkage analysis in four F2 families segregating for Choc and WT larval coloration phenotypes (Figure $2 \mathrm{c}$ and Supplementary Table S2). An intron length polymorphism in this $B$. anynana gene was identified in the grandparents: the Choc grandparents carried two 1086-bp alleles (small, S), while the WT grandparents carried a 1189-bp (middle, M) and a 1305-bp (large, L) alleles. These were used to genotype the eight $\mathrm{F} 1$ parents and $349 \mathrm{~F} 2$ offspring in the mapping panel. None of the 85 F2 offspring with WT larval coloration $(+/+)$ had the Choc-specific S allele. On the other hand, 109 Choc larvae (putative Choc/Choc homozygotes) carried two S alleles, and 155 Choc larvae (putative Choc/+ heterozygotes) carried one S allele and either $\mathrm{M}$ or L alleles. This perfect co-segregation between the polymorphic marker and larval phenotype indicates that the Choc locus is tightly linked (within $0.29 \mathrm{cM}$, equivalent to fewer than 1 recombination event in 349 meiosis) to EST GE676169 and is likely an allele of the corresponding gene.

\section{The Choc locus encodes a putative pyridoxal phosphate (PLP)-} dependent cysteine sulfinic acid decarboxylase

We obtained the full-length $B$. anynana ortholog of the B. mori gene BGIBMGA010122 (see Materials and methods) and established that its 1452-bp open reading frame encodes a 483 -amino acid protein with a DOPA decarboxylase domain (Figure 3a). This domain belongs to a superfamily of highly conserved domains, the PLP-dependent aspartate aminotransferases. These are characterized by seven conserved pyridoxal $5^{\prime}$-phosphate-binding residues and one catalytic residue lysine (see Figure 3a), which forms the aldimine bond with the coenzyme (John, 1995). Phylogenetic analysis based on the amino acid sequences of the B. anynana enzyme and eleven putative homologs from four other insect species (see Materials and methods for selection details) revealed that it is closely related to predicted proteins of B. mori (BGIBMGA010122 in Silkworm Genome Database), Tribolium castaneum (014177 in BeetleBase; http://beetlebase.org/), and D. melanogaster (CG5618 in FlyBase). The function of these proteins, which are clearly separated from insect Black and Gad1 enzymes (Figure 3b), has not been shown experimentally, but D. melanogaster CG5618 is predicted (cf. FlyBase) to be a PLP-dependent enzyme with cysteine sulfinic acid decarboxylase activity, or CSAD (enzyme nomenclature code EC 4.1.1.29). This suggests that its B. anynana ortholog, hereafter referred to as $B a_{-} C S A D$, can perform a similar role.

PLP-dependent enzymes catalyse a wide variety of biochemical reactions (for example, decarboxylation or transamination) that almost exclusively involve amino acids (Percudani and Peracchi, 2003; Mozzarelli and Bettati, 2006). Approximately $0.5 \%$ of genes in any eukaryotic genome encode PLP-dependent enzymes, and over 100 distinct enzymatic activities are carried by these proteins (Christen and Mehta, 2001). Well-studied enzymes of this group include DOPA decarboxylase, which catalyzes the decarboxylation of tyrosine, and glutamate and aspartate decarboxylases (Richardson et al., 2010). CSAD enzymes have been implicated in taurine biosynthesis in mammals (Guion-Rain et al., 1975), but, to our knowledge, have not been associated with pigmentation or any other biological process in insects. Our linkage mapping puts the Choc allele within $0.29 \mathrm{cM}$ of the putative $B a \_C S A D$. The relationship between genetic and physical maps has not been determined for $B$. anynana, but in the highly syntenic (Beldade et al., 2009) reference genome of $B$. mori $0.29 \mathrm{cM}$ is estimated to correspond to $87-174 \mathrm{~Kb}$ (Sato et al., 2008). Within $174 \mathrm{~Kb}$ to the left and to the right of B. mori BGIBMGA010122 in nscaf2986 there are 12 predicted genes (cf. Silkworm Genome Database), none with described role in melanogenesis (see Supplementary Table S4).

Many cases of larval melanism have been documented in association with thermoregulation and high larval densities (Goulson, 1994; Hazel, 2002; Noor et al., 2008), yet little is known about the genetic basis of this phenotype. Our study suggests a novel candidate locus for larval melanism and open up new prospects for further analysis of the role of CSADs in the adaptive evolution of insect coloration.

\section{Regulatory and coding sequence variation of $B a_{-} C S A D$}

To assess whether the causal mutation occurred in the coding or regulatory sequence of $B a_{-} C S A D$, we looked for differences in proteincoding sequence and in expression levels between Choc and WT individuals. The complete coding sequence of the gene was analyzed in four WT and four Choc sibling offspring of a single cross between a Choc/ + male and a $+/+$ female. No indels causing a frameshift in the open reading frame, or SNPs resulting in a premature stop codon were found in the mutant. Of the five SNPs identified, three are synonymous and two adjacent SNPs (AA in the Choc allele vs CC in the WT at positions $1436+1437 \mathrm{bp}$ in JN003848) cause a threonine to lysine substitution in position 479 of the 483 -aa predicted protein, which is outside the functional domain (Figure 3a). However, this substitution might be functionally relevant because amino acid changes outside catalytic sites can still affect enzymatic function, for example, by changing the structure of the protein (Freeman et al., 2011).

Expression levels of $B a \_C S A D$ and of seven genes with known function in insect pigmentation were examined by semi-quantitative PCR. This analysis targeted four stages around the last larval molt (that is, when cuticular pigmentation takes place) and during the first 2 days of the last larval instar (that is, when the differences between phenotypes are the most obvious), in replicate individuals with either 
WT or Choc coloration (Figure 4). The genes pale, Ddc, black, tan, yellow and ebony encode enzymes of the melanin biosynthesis pathway, whereas the product of purple is involved in the biosynthesis of a cofactor for Pale (see Wittkopp et al., 2003, Futahashi et al., 2010, and references therein). No variation was observed between the phenotypes or stages in the expression levels of the control gene FK506 and pigmentation genes purple, pale, Ddc, black, and tan. Expression of yellow was relatively high around the molt (consistent with what has been described in other butterflies; Futahashi et al., 2010), whereas ebony (mapped to LG 26 in the syntenic genome of B. mori) was expressed at relatively low levels across all stages, and downregulated in late 4th instar Choc individuals (Supplementary Figure S1), suggesting a regulatory feedback with allelic variation at $B a_{-} C S A D$ (the locus the Choc phenotype maps to).

The candidate gene Ba_CSAD was expressed in 21 out of 24 WT individuals, and in only 8 out of 24 Choc larvae examined (Figure $4 \mathrm{c}$ and Supplementary Figure S1). The contrast between the WT and mutant phenotypes was particularly obvious during the late 4th instar (Ba_CSAD expressed in all six WT and in just one out of six Choc individuals), and on the second day of the 5th instar (transcripts of $B a \_C S A D$ detected in all six WT, but in none of the Choc larvae). The correlation between larval pigmentation phenotype and $B a_{-} C S A D$ expression is not perfect, which could be explained by: (1) discrepancies in 'physiological' and 'chronological' stages (differences in physiological state among larvae within same group could result from our staging method, based on a combination of morphological markers and chronological time), (2) differences between males and females (larval sex was not determined here), and (3) cryptic variation in gene expression (cf. Reed et al., 2007). Taking together our mapping data, that place the locus responsible for the Choc phenotype within $0.29 \mathrm{cM}$ of $B a_{-} C S A D$, and these differences in expression of the same gene between WT and Choc larvae, suggests that a mutation in $B a \_C S A D$ is causing the darkening of the larval cuticle in the B. anynana Choc individuals. With all data taken together, it seems unlikely that the expression difference we see for Ba_CSAD between Choc and WT is not related to the difference in pigmentation, or that it is controlled by some trans-acting factor diverged between the two lab populations.

\section{Concluding remarks}

Analysis of laboratory mutants has proven to be useful in evo-devo studies of animal pigmentation (Protas and Patel, 2008). We investigate two melanic mutations in the butterfly model, $B$. anynana, and provide several lines of evidence suggesting that allelic variation at the $B a \_C S A D$ locus could be responsible for the larval color phenotype in the Choc mutant. First, the Ba_CSAD and Choc loci are mapped to within $0.29 \mathrm{cM}$ of each other in a region that does not contain any other pigmentation candidate genes (cf. the analysis of the syntenic, fully sequenced genome of the silkworm; see Supplementary Table S4). Second, we identified differences between WT and mutant individuals, both in the coding sequence (an amino acid substitution outside the catalytic domain) and in expression levels (downregulation around the last larval molt) of $B a_{-} C S A D$. Additional studies are required to analyze the function (for example, substrate specificity) of this enzyme, to characterize the putative effects of the amino acid changes we detected, and to identify the nucleotide changes responsible for expression differences.

We also show that the larval and adult melanic phenotypes are due to alleles at two different, independently segregating loci, and that the latter is not due to a mutation in the candidate gene Ba_black whose orthologs have been implicated in similar phenotypes in other insects.
Mutant alleles such as Choc and $\mathrm{mln}$ provide the opportunity to explore the genetic basis of melanism, and to investigate whether the loci identified in lab mutants are important in micro- and macroevolutionary processes in nature (Haag and True, 2001). Furthermore, mutations with such a stage-restricted effect on pigmentation indicate that larval and adult coloration are, to some extent, regulated independently, and offer a chance to explore experimentally this type of developmental uncoupling.

\section{DATA ARCHIVING}

Sequence data have been submitted to GenBank: accession numbers JN003848-JN003850. Phenotype and genotype data from the linkage mapping experiments have been deposited in the Dryad repository: doi:10.5061/dryad.1925530c.

\section{CONFLICT OF INTEREST}

The authors declare no conflict of interest.

\section{ACKNOWLEDGEMENTS}

We thank the students Ana Marcelino (for cloning of ebony and genotyping of Ba_black), Eline Reijmerink (for crosses between Choc and mln mutants) and Paul Brakefield for establishing the mutant stocks and for discussions about them. This work was supported by grants to PB from the Portuguese Foundation for Science and Technology (FCT, PTDC/BIA-BDE/099808/2008) and the Dutch Science Organization (NWO, VIDI 864.08.010).

Allen CE, Beldade P, Zwaan BJ, Brakefield PM (2008). Differences in the selection response of serially repeated color pattern characters: standing variation, development, and evolution. BMC Evol Biol 8: 94

Arakane Y, Lomakin J, Beeman RW, Muthukrishnan S, Gehrke SH, Kanost MR et al. (2009). Molecular and functional analyses of amino acid decarboxylases involved in cuticle tanning in Tribolium castaneum. J Biol Chem 284: 16584-16594.

Bear A, Simons A, Westerman E, Monteiro A (2010). The genetic, morphological, and physiological characterization of a dark larval cuticle mutation in the butterfly, Bicyclus anynana. PLoS One 5: e11563.

Beldade P, Brakefield PM (2002). The genetics and evo-devo of butterfly wing patterns. Nat Rev Genet 3: 442-452.

Beldade P, Brakefield PM, Long AD (2005). Generating phenotypic variation: prospects from "evo-devo" research on Bicyclus anynana wing patterns. Evol Dev 7: 101-107.

Beldade P, French V, Brakefield PM (2008). Developmental and genetic mechanisms for evolutionary diversification of serial repeats: eyespot size in Bicyclus anynana butterflies. J Exp Zool B Mol Dev Evol 310: 191-201.

Beldade P, Koops K, Brakefield PM (2002). Developmental constraints versus flexibility in morphological evolution. Nature 416: 844-847.

Beldade P, Mateus ARA, Keller RA (2011). Evolution and molecular mechanisms of adaptive developmental plasticity. Mol Ecol 20: 1347-1363.

Beldade P, Rudd S, Gruber JD, Long AD (2006). A wing expressed sequence tag resource for Bicyclus anynana butterflies, an evo-devo model. BMC Genomics 7: 130.

Beldade P, Saenko SV, Pul N, Long AD (2009). A gene-based linkage map for Bicyclus anynana butterflies allows for a comprehensive analysis of synteny with the lepidopteran reference genome. PLoS Genet 5: e1000366.

Brakefield P, Beldade P, Zwaan BJ (2009). The African butterfly Bicyclus anynana: a model for evolutionary genetics and evolutionary developmental biology. In: Behringer RR, Johnson AD, Krumlauf RE (eds). Emerging Model Organisms: A Laboratory Manual. Cold Spring Harbor Laboratory Press Vol.1, Chapter 12.

Caro T (2005). The adaptive significance of coloration in mammals. BioScience 55: 125-136.

Christen P, Mehta PK (2001). From cofactor to enzymes. The molecular evolution of pyridoxal-5'-phosphate-dependent enzymes. Chem Rec 1: 436-447.

Conceição IC, Long AD, Gruber JD, Beldade P (2011). Genomic sequence around butterfly wing development genes: annotation and comparative analysis. PLoS One 6: e23778.

Eizirik E, Yuhki N, Johnson WE, Menotti-Raymond M, Hannah SS, O'Brien SJ (2003). Molecular genetics and evolution of melanism in the cat family. Curr Biol 13: 448-453. Freeman AM, Mole BM, Silversmith RE, Bourret RB (2011). Action at a distance: amino acid substitutions that affect binding of the phosphorylated $\mathrm{CheY}$ response regulator and catalysis of dephosphorylation can be far from the CheZ phosphatase active site. J Bacteriol 193: 4709-4718.

Futahashi R, Banno Y, Fujiwara H (2010). Caterpillar color patterns are determined by a two-phase melanin gene prepatterning process: new evidence from tan and laccase2. Evol Dev 12: 157-167. 
Futahashi R, Fujiwara H (2005). Melanin-synthesis enzymes coregulate stage-specific larval cuticular markings in the swallowtail butterfly, Papilio xuthus. Dev Genes Evol 215: 519-529.

Futahashi R, Sato J, Meng Y, Okamoto S, Daimon T, Yamamoto K et al. (2008). yellow and ebony are the responsible genes for the larval color mutants of the silkworm Bombyx mori. Genetics 180: 1995-2005.

Gompel N, Prud'homme B, Wittkopp PJ, Kassner VA, Carroll SB (2005). Change caught on the wing: cis-regulatory evolution and the origin of pigment patterns in Drosophila. Nature 433: 481-487.

Goulson D (1994). Determination of larval melanization in the moth, Mamestra brassicae, and the role of melanin in thermoregulation. Heredity 73: 471-479.

Gross JB, Borowsky R, Tabin CJ (2009). A novel role for Mc1r in the parallel evolution of depigmentation in independent populations of the cavefish Astyanax mexicanus. PLoS Genet 5: e1000326.

Guion-Rain MC, Portemer C, Chatagner F (1975). Rat liver cysteine sulfinate decarboxylase: purification, new appraisal of the molecular weight and determination of catalytic properties. Biochim Biophys Acta 384: 265-276.

Haag ES, True JR (2001). From mutants to mechanisms? Assessing the candidate gene paradigm in evolutionary biology. Evolution 55: 1077-1084.

Hazel WN (2002). The environmental and genetic control of seasonal polyphenism in larval color and its adaptive significance in a swallowtail butterfly. Evolution 56 342-348.

John RA (1995). Pyridoxal phosphate-dependent enzymes. Biochim Biophys Acta 1248 81-96.

Jones M, Rakes L, Yochum M, Dunn G, Wurster S, Kinney K et al. (2007). The proximate control of pupal color in swallowtail butterflies: implications for the evolution of environmentally cued pupal color in butterflies (Lepidoptera: Papilionidae). J Insect Physiol 53: 40-46.

Koch PB, Behnecke B, Weigmann-Lenz M, Ffrench-Constant RH (2000). Insect pigmentation: activities of beta-alanyldopamine synthase in wing color patterns of wild-type and melanic mutant swallowtail butterfly Papilio glaucus. Pigment Cell Res (Suppl)8: 54-58.

Kramer KJ, Morgan TD, Hopkins TL, Roseland CR, Aso Y, Beeman RW et al. (1984). Cateholamines and $\beta$-alanine in the red flour beetle, Tribolium castaneum - roles in cuticle sclerotization and melanization. Insect Biochem 14: 293-298.

Manceau M, Domingues VS, Mallarino R, Hoekstra HE (2011). The developmental role of Agouti in color pattern evolution. Science 331: 1062-1065.

Mills MG, Nuckels RJ, Parichy DM (2007). Deconstructing evolution of adult phenotypes: genetic analyses of kit reveal homology and evolutionary novelty during adult pigment pattern development of Danio fishes. Development 134: 1081-1090.

Mills MG, Patterson LB (2009). Not just black and white: pigment pattern development and evolution in vertebrates. Semin Cell Dev Biol 20: 72-81.

Mozzarelli A, Bettati S (2006). Exploring the pyridoxal 5'-phosphate-dependent enzymes. Chem Rec 6: 275-287.

Nachman MW, Hoekstra HE, D'Agostino SL (2003). The genetic basis of adaptive melanism in pocket mice. Proc Natl Acad Sci USA 100: 5268-5273.

Noor MA, Parnell RS, Grant BS (2008). A reversible color polyphenism in American peppered moth (Biston betularia cognataria) caterpillars. PLoS One 3: e3142.

Oliver JC, Robertson KA, Monteiro A (2009). Accommodating natural and sexual selection in butterfly wing pattern evolution. Proc Biol Sci 276: 2369-2375.

Percudani R, Peracchi A (2003). A genomic overview of pyridoxal-phosphate-dependent enzymes. EMBO reports 4: 850-854.
Phillips AM, Smart R, Strauss R, Brembs B, Kelly LE (2005). The Drosophila black enigma: the molecular and behavioural characterization of the black1 mutant allele. Gene 351: 131-142.

Pijpe J, Pul N, van Duijn S, Brakefield PM, Zwaan BJ (2011). Changed gene expression for candidate ageing genes in long-lived Bicyclus anynana butterflies. Exp Gerontol 46: 426-434.

Protas ME, Patel NH (2008). Evolution of coloration patterns. Annu Rev Cell Dev Biol 24 : 425-446.

Protas ME, Trontelj P, Patel NH (2011). Genetic basis of eye and pigment loss in the cave crustacean, Asellus aquaticus. Proc Natl Acad Sci USA 108: 57025707.

Reed RD, Chen PH, Frederik Nijhout H (2007). Cryptic variation in butterfly eyespot development: the importance of sample size in gene expression studies. Evol Dev 9: 2-9.

Richardson G, Ding H, Rocheleau T, Mayhew G, Reddy E, Han Q et al. (2010). An examination of aspartate decarboxylase and glutamate decarboxylase activity in mosquitoes. Mol Biol Rep 37: 3199-3205.

Rosenblum EB, Römpler H, Schöneberg T, Hoekstra HE (2010). Molecular and functional basis of phenotypic convergence in white lizards at White Sands. Proc Natl Acad Sci USA 107: 2113-2117.

Saenko SV, Brakefield PM, Beldade P (2010). Single locus affects embryonic segment polarity and multiple aspects of an adult evolutionary novelty. BMC Biol 8: 111 .

Saenko SV, French V, Brakefield PM, Beldade P (2008). Conserved developmental processes and the formation of evolutionary novelties: examples from butterfly wings. Philos Trans R Soc Lond B Biol Sci 363: 1549-1555.

Saenko SV, Marialva MS, Beldade P (2011). Involvement of the conserved Hox gene Antennapedia in the development and evolution of a novel trait. EvoDevo 2: 9.

Sato K, Matsunaga TM, Futahashi R, Kojima T, Mita K, Banno Y et al. (2008). Positional cloning of a Bombyx wingless locus flugellos ( $f$ ) reveals a crucial role for fringe that is specific for wing morphogenesis. Genetics 179: 875-885.

True JR (2003). Insect melanism: the molecules matter. Trends Ecol Evol 18: 640-647.

True JR, Yeh SD, Hovemann BT, Kemme T, Meinertzhagen IA, Edwards TN et al. (2005). Drosophila tan encodes a novel hydrolase required in pigmentation and vision. PLOS Genet 1: e63.

van't Hof AE, Edmonds N, Dalíková M, Marec F, Saccheri IJ (2011). Industrial melanism in british peppered moths has a singular and recent mutational origin. Science 332 958-960.

van't Hof AE, Saccheri IJ (2010). Industrial melanism in the peppered moth is not associated with genetic variation in canonical melanisation gene candidates. PLoS One 5: e10889.

Weatherbee SD, Nijhout HF, Grunert LW, Halder G, Galant R, Selegue J et al. (1999). Ultrabithorax function in butterfly wings and the evolution of insect wing patterns. Curr Biol 9: 109-115.

Wittkopp PJ, Beldade P (2009). Development and evolution of insect pigmentation: genetic mechanisms and the potential consequences of pleiotropy. Semin Cell Dev Biol 20: 65-71.

Wittkopp PJ, Carroll SB, Kopp A (2003). Evolution in black and white: genetic control of pigment patterns in Drosophila. Trends Genet 19: 495-504.

Wittkopp PJ, Vaccaro K, Carroll SB (2002). Evolution of yellow gene regulation and pigmentation in Drosophila. Curr Biol 12: 1547-1556.

Zhan S, Guo Q, Li M, Li M, Li J, Miao X et al. (2010). Disruption of an N-acetyltransferase gene in the silkworm reveals a novel role in pigmentation. Development 137: 4083-4090.

Supplementary Information accompanies the paper on Heredity website (http://www.nature.com/hdy) 